\title{
Poor cardiovascular health is associated with subclinical atherosclerosis in apparently healthy sub-Saharan African populations: an H3Africa AWI-Gen study
}

Engelbert A. Nonterah ${ }^{1,2^{*}}$ (D), Nigel J. Crowther ${ }^{3}$, Abraham Oduro ${ }^{1}$, Godfred Agongo ${ }^{1}$, Lisa K. Micklesfield ${ }^{4}$, Palwendé R. Boua ${ }^{5}$, Solomon S. R. Choma ${ }^{6}$, Shukri F. Mohamed ${ }^{7}$, Herman Sorgho ${ }^{5}$, Stephen M. Tollman', Shane A. Norris ${ }^{4}$, Frederick J. Raal ${ }^{9}$, Diederick E. Grobbee ${ }^{2}$, Michelé Ramsay ${ }^{10}$, Michiel L. Bots ${ }^{2}$, Kerstin Klipstein-Grobusch ${ }^{2,11}$ as part of the H3Africa AWI-Gen study

\begin{abstract}
Background: The cardiovascular health index (CVHI) introduced by the American Heart Association is a valid, accessible, simple, and translatable metric for monitoring cardiovascular health in a population. Components of the $\mathrm{CVHI}$ include the following seven cardiovascular risk factors (often captured as life's simple 7): smoking, dietary intake, physical activity, body mass index, blood pressure, glucose, and total cholesterol. We sought to expand the evidence for its utility to under-studied populations in sub-Saharan Africa, by determining its association with common carotid intima-media thickness (CIMT).

Methods: We conducted a cross-sectional study involving 9011 participants drawn from Burkina Faso, Ghana, Kenya, and South Africa. We assessed established classical cardiovascular risk factors and measured carotid intimamedia thickness of the left and right common carotid arteries using B-mode ultrasonography. Adjusted multilevel mixed-effect linear regression was used to determine the association of CVHI with common CIMT. In the combined population, an individual participant data meta-analyses random-effects was used to conduct pooled comparative sub-group analyses for differences between countries, sex, and socio-economic status.

(Continued on next page)
\end{abstract}

\footnotetext{
* Correspondence: engelbert.nonterah@navrongo-hrc.org

${ }^{1}$ Clinical Sciences Department, Navrongo Health Research Centre, Ghana

Health Service, Navrongo, Ghana

${ }^{2}$ Julius Global Health, Julius Center for Health Sciences and Primary Care,

University Medical Center Utrecht, Utrecht University, Utrecht, the Netherlands

Full list of author information is available at the end of the article
}

(c) The Author(s). 2021 Open Access This article is licensed under a Creative Commons Attribution 4.0 International License, which permits use, sharing, adaptation, distribution and reproduction in any medium or format, as long as you give appropriate credit to the original author(s) and the source, provide a link to the Creative Commons licence, and indicate if changes were made. The images or other third party material in this article are included in the article's Creative Commons licence, unless indicated otherwise in a credit line to the material. If material is not included in the article's Creative Commons licence and your intended use is not permitted by statutory regulation or exceeds the permitted use, you will need to obtain permission directly from the copyright holder. To view a copy of this licence, visit http://creativecommons.org/licenses/by/4.0/ The Creative Commons Public Domain Dedication waiver (http://creativecommons.org/publicdomain/zero/1.0/) applies to the data made available in this article, unless otherwise stated in a credit line to the data. 


\begin{abstract}
(Continued from previous page)
Results: The mean age of the study population was $51 \pm 7$ years and $51 \%$ were women, with a mean common CIMT of $637 \pm 117 \mu \mathrm{m}$ and $\mathrm{CVHI}$ score of $10.3 \pm 2.0$. Inverse associations were found between CVHI and common CIMT ( $\beta$-coefficients [95\% confidence interval]: Burkina Faso, $-6.51[-9.83,-3.20] \mu \mathrm{m}$; Ghana, $-5.42[-8.90,-1.95]$; Kenya, $-6.58[-9.05,-4.10]$; and South Africa, $-7.85[-9.65,-6.05])$. Inverse relations were observed for women $(-4.44[-6.23,-2.65])$ and men $(-6.27[-7.91,-4.64])$ in the pooled sample. Smoking $(p<0.001)$, physical activity $(p<0.001)$, and hyperglycemia $(p<0.001)$ were related to CIMT in women only, while blood pressure and obesity were related to CIMT in both women and men $(p<0.001)$.

Conclusion: This large pan-African population study demonstrates that CVHI is a strong marker of subclinical atherosclerosis, measured by common CIMT and importantly demonstrates that primary prevention of atherosclerotic cardiovascular disease in this understudied population should target physical activity, smoking, obesity, hypertension, and hyperglycemia.
\end{abstract}

Keywords: Cardiovascular health index, Carotid intima-media thickness, Subclinical atherosclerosis, Sub-Saharan Africa, Primary prevention, Cardiovascular diseases, Screening, Understudied populations

\section{Background}

Cardiovascular disease (CVD) is a leading cause of global deaths $[1,2]$ raising huge public health concerns. SubSaharan Africa (SSA) is experiencing a rising burden of non-communicable diseases including CVD [2], alongside a reduction in the prevalence of infectious diseases [3]. Increasing urbanization, changing lifestyles, and population aging are probably accounting for the surge in the prevalence of CVD in SSA [3, 4]. This presents a challenge to the health systems of African countries and requires a pragmatic approach to the prevention of CVD. Where risk equations such as Framingham [5] and Pooled Cohort Equations (PCE) [6] have served as valuable tools in assessing the risk of CVD globally, these have inherent limitations especially when applied to an African population [7]. The American Heart Association and the American Stroke Association (AHA/ASA) developed the Cardiovascular Health Index (CVHI) as a new public health metric for assessing and monitoring cardiovascular health [8-11]. The components of the CVHI often referred to as life's simple seven include smoking, dietary intake, physical activity, body mass index (BMI), blood pressure, glucose, and total cholesterol.

Due to the lack of data on incident CVD events, carotid-intima media thickness (CIMT) is often used as an alternate marker of CVD risk. Increased CIMT has been shown to be related to several risk factors for CVD and CVD risk globally $[12,13]$ including SSA populations [14].

The CVHI metric is proven to be related to CIMT, a marker of subclinical atherosclerosis [15], and coronary artery calcification [16] in both Caucasian and Asian populations [17]. In Africa, the association of CVHI with subclinical atherosclerosis has only been reported in a relatively small study of HIV-positive $(n=105)$ and noninfected $(n=100)$ individuals [18] in Uganda and recently in a rural setting in Limpopo, South Africa [19]. Expanding the evidence to a larger pan-African population would help to clarify whether the CVHI can be used as a simple screening tool for cardiovascular health within the existing health systems of resource-constrained SSA countries. Therefore, we set out to assess the association between cardiovascular health, using CVHI, and subclinical atherosclerosis in a large understudied African population living in SSA.

\section{Methods}

\section{Study design and population}

The H3Africa Africa-Wits-INDEPTH (www. indepthnetwork.org) partnership for genomic studies (AWIGen) project is a population-based cross-sectional study. Participants included older adult women and men aged 4060 years drawn from six sites in four SSA countries [20, 21].

Five of the sites were INDEPTH-Network (International Network for the Demographic Evaluation of Populations and Their Health) Health and Demographic Surveillance Study (HDSS) sites namely Navrongo HDSS in Ghana, Nanoro HDSS in Burkina Faso, Nairobi urban HDSS in Kenya, and Agincourt and DIMAMO HDSS's in South Africa. The sixth site was the South African MRC/University of the Witwatersrand Developmental Pathways to Health Research Unit (DPHRU) in Soweto, South Africa. Participants were randomly sampled from existing sampling frames in the various recruitment sites. Those who reported history of cardiovascular diseases (myocardial infarction, stroke) and chronic kidney disease were excluded from the analyses. Pregnant women, closely related individuals (first degree relatives), and those residents in the communities for less than 10 years were excluded.

Independent variable: cardiovascular health index (CVHI) The components of the CVHI as defined by AHA/ASA include blood pressure, fasting glucose, total cholesterol, body mass index, physical activity, diet, and smoking [8]. 
Each CVHI component was given a point score of 0,1 , or 2 to represent poor, intermediate, and ideal health respectively. Details of how the categories were defined are presented in Additional file 1: Table S1. The AHA recommended cutoffs are consistent with recommendations from the World Health Organization [22, 23] and other organizations [24]. These cutoffs have recently been used in a study in rural South Africa [19]. An overall CVHI score was computed as the sum of all 7 CVHI components, ranging from 0 to 14 . We stratified participants into 3 mutually exclusive categories based on overall CVHI score: Ideal cardiovascular health $(\mathrm{iCVH})$ defined as 12-14 points, intermediate cardiovascular health 8-11 points, and poor cardiovascular health $0-7$ points [25]. Details of how the various components of CVHI were measured and defined in the AWI-Gen study have been published elsewhere [21].

Briefly, the history of smoking tobacco products was self-reported and defined as never smoked, previous smoker, and current smoker. Physical activity was measured as the level of moderate-to-vigorous physical activity (MVPA) from self-reported data on occupation, travel-related, and leisure time physical activity. Diet was measured as the self-reported daily number of servings of fruits and vegetables. Weight was measured to the nearest $0.1 \mathrm{~kg}$ using a digital $200 \mathrm{~kg}$ capacity scale (Kendon Medical, South Africa). Standing height to the nearest millimeter was measured using a Harpenden digital stadiometer (Holtain, Wales). The BMI was then calculated as weight divided by height squared in kilograms per square meter. Blood pressure was measured from the left arm of the participant, seated with the arm at the level of the heart and having rested 5-10 min. Three readings were taken with the first value discarded and an average of the second and third computed as blood pressure. Venous blood was taken with the participant having fasted overnight, and this sample was used to measure glucose and total cholesterol using colorimetric assays in a Randox Plus clinical chemistry analyzer (Crumlin, UK). The coefficient of variation of the laboratory measurements for glucose was $2.3 \%$ and $1.5 \%$ for total cholesterol. All analyses were performed in a centralized laboratory at the DPHRU in Soweto according to good laboratory practice and with external monitoring for quality control.

\section{Dependent variable: common carotid atherosclerosis}

Carotid intima-media thickness was used as a marker of sub-clinical atherosclerosis. A carotid B-mode ultrasound scan (GE Healthcare, USA) with a $12 \mathrm{~L}-\mathrm{RS}$ straight probe was used to measure the CIMT of the right and left common carotid artery (CCA). Details of the measurement procedure used in this population are described elsewhere $[14,21]$. Briefly, the right and left intima-media thickness of the common carotid arteries (CCA) were measured at a single angle of 45 degree tilt with the participant lying supine and neck supported with a pillow. A mean of the right and left common CIMT (in micrometers) was computed as the outcome variable.

\section{Potential confounders and effect modifiers}

Age was self-reported or calculated from reported date of birth while sex was reported at the time of recruitment. Household socio-economic status (SES) was determined based on household assets and quintiles calculated according to the practice implemented by the Demographic and Health Surveys (DHS) Program (http://www.dhsprogram. com/topics/wealth-index/Wealth-Index-Construction. $\mathrm{cfm})$. A principal component analysis of the listed household assets is conducted and predicting factor scores generated from these analyses. The predicted factor scores are then categorized into quintiles for each of the participating sites separately [21]. Educational status was by asking the number years spent in school. Educational status was ascertained by asking the highest level of education (no formal education, primary, secondary or tertiary education). This was subsequently categorized as no formal education and yes formal education (primary, secondary and tertiary education). This took into consideration the number of years in each country at the different levels of school. HIV infection was self-reported in Burkina Faso and Ghana and testing was not offered due to the very low prevalence. Kenya and South Africa used locally available, government-approved rapid-test to conduct voluntary HIV rapid test. Antiretroviral therapy (ART) use was self-reported across all sites.

\section{Statistical analyses}

All data analyses were conducted using STATA version 14.2 (Statacorp, College Station, TX, USA). We used descriptive and inferential statistics to summarize our data. Using Epanechnikov kernel function in STATA, density curves were plotted to determine the distribution of the CVHI score and common CIMT for the various countries represented in the AWI-Gen study (Additional file 2: Figs. S1 and S2). Continuous variables are reported as mean and standard deviation $( \pm \mathrm{SD})$ due to approximate normal distribution and categorical variables as sample number with respective percentages. The differences in prevalence of overall cardiovascular health between women and men were examined using Pearson's chisquared test.

As the participants were resident in four different countries, we used multilevel mixed-effects generalized linear models to determine the associations of CVHI score with common CIMT. A variance co-variance method was used to obtain robust standard errors. Four progressively adjusted models were conducted as follows: model 1 was 
adjusted for age and sex; model 2 was adjusted for age, sex, level of education, and household socio-economic status; in model 3, we adjusted for all the model 2 variables in addition to HIV and ART use. The fixed effects portion of the model was considered to vary according to country and hence country was specified as the random effect part of the mixed effect model. Separate models were fitted for the combined populations, women and men and countries.

The country-specific models were fitted sequentially following the pattern explained above using adjusted linear regression models. Multilevel mixed-effects generalized linear models were conducted for South Africa which contained three different sites (site as the random effect). In the combined population, an individual participant data (IPD) meta-analyses was conducted. Pooled comparative sub-group analyses included comparison by country, sex, and household SES. Our sub-group analyses included household SES since it has previously been linked to poor health outcomes [26] and atherosclerosis [27]. Forest plots were subsequently plotted and weighted by sample size to avoid biased estimates (and for ease of comparison) and by default sample size instead of study weights appear on the plots. In addition, line plots of the predicted linear association between CVHI score and CIMT were drawn and presented in supplementary material (Additional file 2: Figs. S3 and S4). To determine the discriminatory power of each CVHI component metric to predict common CIMT, we generated dummy variables for the 3 levels of each CVHI metric using "poor" as the reference. We then conducted adjusted multilevel mixed effects linear regression analyses of the dummy variables, with adjustment for age, sex, educational status, and household SES in the pooled sample and for women and men separately. A two-tailed $p<0.05$ was considered statistically significant in all inferential analyses.

\section{Results}

After excluding participants (7.5\%) with missing data, 9011 adults from Burkina Faso, Ghana, Kenya, and South Africa were included in these analyses. Basic characteristics are presented in Table 1. Fifty one percent of the study population was women and the average age was $51 \pm 7$ years. Physical activity levels were high (higher mean moderate-to-vigorous physical activity minutes/week) in the populations from the two rural sites of West Africa. The average fruit and vegetable intake in the combined AWI-Gen population was $3 \pm 2$ servings per day. Mean BMI and systolic and diastolic blood pressure were highest in South Africa and Kenya and lowest in Ghana and Burkina Faso (Table 1).

Table 1 Socio-demographic characteristics of the AWI-Gen participants per research site

\begin{tabular}{|c|c|c|c|c|c|}
\hline Characteristics & $\begin{array}{l}\text { Total population } \\
N=9011\end{array}$ & $\begin{array}{l}\text { Burkina Faso } \\
n=2074\end{array}$ & $\begin{array}{l}\text { Ghana } \\
n=1729\end{array}$ & $\begin{array}{l}\text { Kenya } \\
n=1913\end{array}$ & $\begin{array}{l}\text { South Africa } \\
n=3295\end{array}$ \\
\hline Women & $4948(50.8)$ & 1035 (49.6) & $936(54.1)$ & $1067(54.1)$ & $1910(48.2)$ \\
\hline Age in years & $50.9 \pm 6.9$ & $49.8 \pm 5.9$ & $51.1 \pm 5.8$ & $48.8 \pm 5.8$ & $52.5 \pm 8.0$ \\
\hline Formal education in yes/no & $6675(63.8)$ & $353(17.0)$ & $594(29.6)$ & $1795(92.4)$ & $3933(88.5)$ \\
\hline *Household SES & $10.1 \pm 4.1$ & $11.3 \pm 3.9$ & $9.50 \pm 4.6$ & $11.1 \pm 3.3$ & $9.1 \pm 4.0$ \\
\hline \multicolumn{6}{|l|}{ Household SES in quintiles } \\
\hline Q1 (poorest) & $336(16.2)$ & $372(18.5)$ & $235(12.1)$ & $605(13.4)$ & $1548(14.7)$ \\
\hline Q2 (poorer) & $404(19.4)$ & $360(17.9)$ & $432(22.3)$ & $1100(24.4)$ & $2296(21.8)$ \\
\hline Q3 (poor) & $405(19.5)$ & $387(19.2)$ & $450(23.2)$ & $655(14.6)$ & $1897(18.0)$ \\
\hline Q4 (less poor) & $386(18.6)$ & $473(23.5)$ & $396(20.4)$ & $949(21.1)$ & $2204(20.9)$ \\
\hline Q5 (least poor) & $546(26.3)$ & $421(20.9)$ & $429(22.1)$ & $1194(26.5)$ & $2590(24.6)$ \\
\hline MVPA in minutes/weeks & $1573 \pm 1550$ & $2077 \pm 1590$ & $2036 \pm 1707$ & $1749 \pm 1732$ & $1074 \pm 1189$ \\
\hline Servings of fruits and vegetables/day & $2.9 \pm 2.0$ & $1.8 \pm 0.9$ & $3.4 \pm 1.5$ & $4.11 \pm 2.5$ & $2.23 \pm 1.7$ \\
\hline $\mathrm{BMl}$ in $\mathrm{kg} / \mathrm{m}^{2}$ & $25.0 \pm 6.8$ & $20.9 \pm 3.4$ & $21.60 \pm 3.6$ & $25.40 \pm 5.8$ & $28.22 \pm 7.6$ \\
\hline $\mathrm{SBP}$ in $\mathrm{mmHg}$ & $124.6 \pm 21.8$ & $115.6 \pm 18.2$ & $123.9 \pm 21.6$ & $120.0 \pm 21.2$ & $130.9 \pm 21.7$ \\
\hline DBP in $\mathrm{mmHg}$ & $79.7 \pm 3.7$ & $73.5 \pm 10.6$ & $76.9 \pm 12.7$ & $78.4 \pm 12.9$ & $84.29 \pm 14.2$ \\
\hline Glucose in mg/dL & $91.3 \pm 29.4$ & $90.9 \pm 22.5$ & $81.8 \pm 13.21$ & $97.8 \pm 36.1$ & $92.5 \pm 33.5$ \\
\hline Total cholesterol in mg/dL & $148 \pm 43.7$ & $132 \pm 36.9$ & $125 \pm 35.8$ & $169 \pm 41.9$ & $159 \pm 43.2$ \\
\hline CVHI (0-14) & $10.4 \pm 2.0$ & $10.8 \pm 1.5$ & $11.4 \pm 1.6$ & $10.7 \pm 1.9$ & $9.3 \pm 2.0$ \\
\hline Common CIMT in $\mu \mathrm{m}$ & $637 \pm 117$ & $664 \pm 118$ & $690 \pm 119$ & $588 \pm 104$ & $620 \pm 106$ \\
\hline
\end{tabular}

Values are $n$ (\%) or mean \pm SD (standard deviation); CVHI cardiovascular health index, CIMT carotid intima-media thickness, BMI body mass index, SBP systolic blood pressure, DBP diastolic blood pressure, SES socio-economic status, Q1 to Q5 wealth quintiles, MVPA moderate-to-vigorous physical activity in minutes per week. Formal education is defined as having attained primary or secondary or tertiary education; *Household SES presented as a mean \pm SD of household assets 
The prevalence of each of the 3 levels of cardiovascular health (poor, intermediate, and ideal) for the total sample, the total sample stratified by sex, and the various countries is presented in Fig. 1. In the combined sample, $\mathrm{iCVH}$ was $31.9 \%$ with women (38.2\%) having a higher prevalence than men (25.6\%). Rural sites in Ghana (50.6\%) and Burkina Faso (44.3\%) had the highest prevalence of $\mathrm{iCVH}$ while the more urban sites in Kenya (37.4\%) and South Africa (11.3\%) had the lowest. The prevalence of the poor, intermediate, and ideal levels of each of the 7 CVHI components, according to sex and country, is presented in Table 2. The majority of participants were in the ideal category for total cholesterol, fasting glucose, physical activity, BMI, and blood pressure with unhealthy diet and smoking placing the majority of participants in the poor category. Burkina Faso and Ghana had more participants in the ideal category for most CVHI components than South Africa and Kenya, except diet and physical activity where Burkina Faso had the least ideal levels compared to other countries.
There were inverse linear associations between CIMT and CVHI in our combined population $(r=-0.064 ; p<$ $0.001)$ and for Burkina Faso $(r=-0.126 ; p<0.001)$, Ghana $(r=-0.085 ; p<0.001)$, Kenya $(r=-0.178 ; p<$ $0.001)$, and South Africa $(r=-0.199 ; p<0.001)$, and these are presented as line plots in Additional file 2: Figs. S3 and S4. In the adjusted multilevel mixed-effects generalized linear regression model for the combined population, a unit increase in the CVHI was associated with - 5.54 $[-6.75,-4.32] \mu \mathrm{m}$ decrease in common CIMT (Fig. 2). The risk of atherosclerosis (high common CIMT) was progressively lower with increasing CVHI in each country, sex, and household SES category in both the combined sample (Fig. 2) and the sex stratified analyses (Figs. 3 and 4). A similar inverse association was observed in women $(-4.44[-6.23,-2.65])$ and in men $(-6.27[-7.98,-4.64])$ (Figs. 3 and 4, respectively).

Sequential adjustments of the regression models are presented in Table 3 and demonstrate minimal effects of adjustment on the $\beta$-coefficients. All models demonstrated

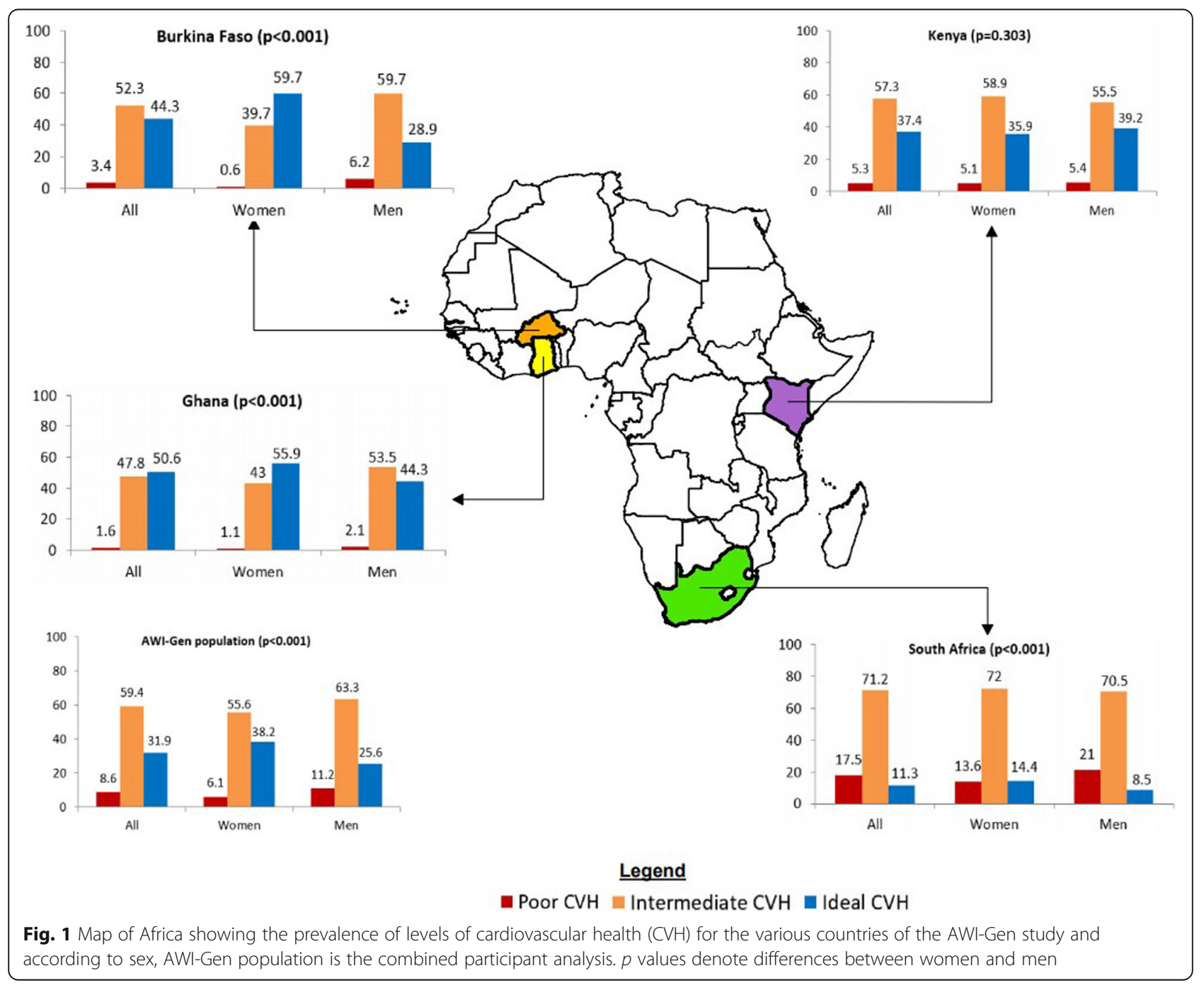


Table 2 The distribution of the poor, intermediate, and ideal levels of cardiovascular health for each of the $7 \mathrm{CVHI}$ metrics in the AWI-Gen sample stratified by sex and country

\begin{tabular}{|c|c|c|c|c|c|c|c|c|}
\hline CVHI metrics & Levels & Combined sample & Women & Men & Burkina Faso & Ghana & Kenya & South Africa \\
\hline \multirow[t]{3}{*}{ Smoking } & Poor & $984(10.9)$ & $103(2.3)$ & $881(19.7)$ & $124(6.0)$ & $190(11.0)$ & $309(16.2)$ & $361(11.0)$ \\
\hline & Intermediate & $1600(17.8)$ & $73(1.6)$ & $1527(34.1)$ & $142(6.8)$ & $355(20.5)$ & $230(12.0)$ & $873(26.5)$ \\
\hline & Ideal & $6427(71.3)$ & $4361(96.1)$ & $2066(46.2)$ & $1808(87.2)$ & $1184(68.5)$ & $1374(71.8)$ & $2061(62.5)$ \\
\hline \multirow[t]{3}{*}{ Physical activity } & Poor & $1355(15.0)$ & $603(13.3)$ & $752(16.8)$ & $402(19.4)$ & $257(14.9)$ & $130(6.8)$ & $566(17.2)$ \\
\hline & Intermediate & $442(4.9)$ & $227(5.0)$ & $215(4.8)$ & $63(3.0)$ & $86(5.0)$ & $129(6.7)$ & $164(5.0)$ \\
\hline & Ideal & $7214(80.1)$ & 3707 (81.7) & 3507 (78.4) & 1609 (77.6) & $1386(80.2)$ & $1654(86.5)$ & $2565(77.8)$ \\
\hline \multirow[t]{3}{*}{ Healthy diet score } & Poor & $4266(56.3)$ & $2359(56.2)$ & $1907(56.5)$ & $1324(82.4)$ & $518(30.0)$ & $578(30.2)$ & $1846(79.4)$ \\
\hline & Intermediate & $1946(25.7)$ & $1070(25.5)$ & $876(26.0)$ & $271(16.9)$ & $778(45.0)$ & $620(32.4)$ & 277 (11.9) \\
\hline & Ideal & $1361(18.0)$ & $770(18.3)$ & $591(17.5)$ & $12(0.7)$ & $433(25.0)$ & $715(37.4)$ & $201(8.6)$ \\
\hline \multirow[t]{3}{*}{ Body mass index } & Poor & $1393(15.5)$ & $1092(24.1)$ & $301(6.7)$ & $36(1.7)$ & $45(2.6)$ & 375 (19.6) & $937(28.4)$ \\
\hline & Intermediate & 1673 (18.6) & $914(20.2)$ & $759(17.0)$ & $177(8.5)$ & $168(9.7)$ & $494(25.8)$ & $834(25.3)$ \\
\hline & Ideal & $5943(66.0)$ & 2529 (55.8) & $3414(76.3)$ & 1861 (89.7) & 1516 (87.7) & $1043(54.6)$ & $1523(46.2)$ \\
\hline \multirow[t]{3}{*}{ Blood pressure } & Poor & $1211(13.4)$ & $548(12.1)$ & $663(14.8)$ & $123(5.9)$ & $210(12.1)$ & $216(11.3)$ & $662(20.1)$ \\
\hline & Intermediate & 2204 (24.5) & $1006(22.2)$ & $1198(26.8)$ & 331 (16.0) & $380(22.0)$ & $445(23.3)$ & $1048(31.8)$ \\
\hline & Ideal & $5596(62.1)$ & $2983(65.7)$ & $2613(58.4)$ & $1620(78.1)$ & 1139 (65.9) & $1252(65.4)$ & $1585(48.1)$ \\
\hline \multirow[t]{3}{*}{ Fasting glucose } & Poor & $513(5.7)$ & $281(6.2)$ & $232(5.2)$ & $77(3.7)$ & $70(4.0)$ & 127 (6.6) & $239(7.3)$ \\
\hline & Intermediate & $1203(13.4)$ & $569(12.5)$ & $634(14.2)$ & $305(14.7)$ & $113(6.5)$ & $354(18.5)$ & $431(13.1)$ \\
\hline & Ideal & 7295 (81.0) & 3687 (81.3) & 3608 (80.6) & 1692 (81.6) & 1546 (89.4) & $1432(74.9)$ & $2625(79.7)$ \\
\hline \multirow[t]{3}{*}{ Total cholesterol } & Poor & 350 (3.9) & $185(4.1)$ & 165 (3.7) & $44(2.1)$ & $10(0.6)$ & $107(5.6)$ & $189(5.7)$ \\
\hline & Intermediate & $805(8.9)$ & $425(9.4)$ & $382(8.5)$ & $97(4.7)$ & $44(2.5)$ & $282(14.6)$ & $382(11.6)$ \\
\hline & Ideal & 7856 (87.2) & 3927 (86.6) & 3929 (87.8) & 1933 (93.2) & 1675 (96.9) & $1524(79.7)$ & $2724(82.7)$ \\
\hline
\end{tabular}

Data presented as $n$ (\%); levels of $\mathrm{CVH}=$ cardiovascular health which is defined as poor (CVHI score of 0-7), intermediate (CVHI score of 8-11), and optimum (CVHI score of 12-14)

a significant inverse association between the CVHI and common CIMT, except in women from Burkina Faso and men from Ghana, where the associations were again negative but with the upper 95\% CI just overlapping zero. In adjusted analyses looking at the contribution of the individual components of CVHI to common CIMT, we observed that blood pressure, BMI, and hyperglycemia were associated with common CIMT in the combined population, while smoking and physical activity were associated with common CIMT in women only (see Table 4).

\section{Discussion}

Results from this large population-based study of middleaged men and women from four countries in sub-Saharan Africa highlight relatively high levels of ideal cardiovascular health. We have also shown a significant inverse linear association between increasing CVH score and common CIMT independent of age, educational status, household socio-economic status, HIV infection, and ART use and prior history of cardiovascular or chronic kidney diseases. To our knowledge, this study is the largest to demonstrate an association between the CVHI metric and subclinical atherosclerosis in an apparently healthy adult population from multiple countries in SSA.
The prevalence of ideal cardiovascular health (iCVH) was $31.9 \%$ in the combined AWI-Gen population, and this was higher than the $6.8 \%$ reported in the multi-ethnic study of atherosclerosis (MESA) conducted among Caucasians, Chinese, African Americans, and Hispanics in the USA [25]. Similarly, data from the Behavioral Risk Factor Surveillance System study which used information obtained telephonically from nearly 1.4 million subjects in the USA reported a much lower prevalence of iCVH (5.1\%) in 2009 than reported in the current study [28]. The iCVH levels among our participants from Ghana was $50.9 \%$ and higher than reported by the Research on Obesity and Diabetes among African Migrants (RODAM) study conducted among Ghanaians residing in rural (25.7\%) and urban $(7.5 \%)$ areas and their counterparts in the diaspora $(<5 \%)$ [29]. Further to this, a systematic review by Peng et al. showed that participants from Africa were likely to have higher proportions of ideal CVH compared to North and South Americans, Asians, Europeans, and the Oceania's [30]. The RODAM study, however, used the comprehensive five dietary components (as described in the original CVHI) to define healthy diet whereas only two components were available for use in the AWI-Gen study. This may therefore result in over classifying AWI- 


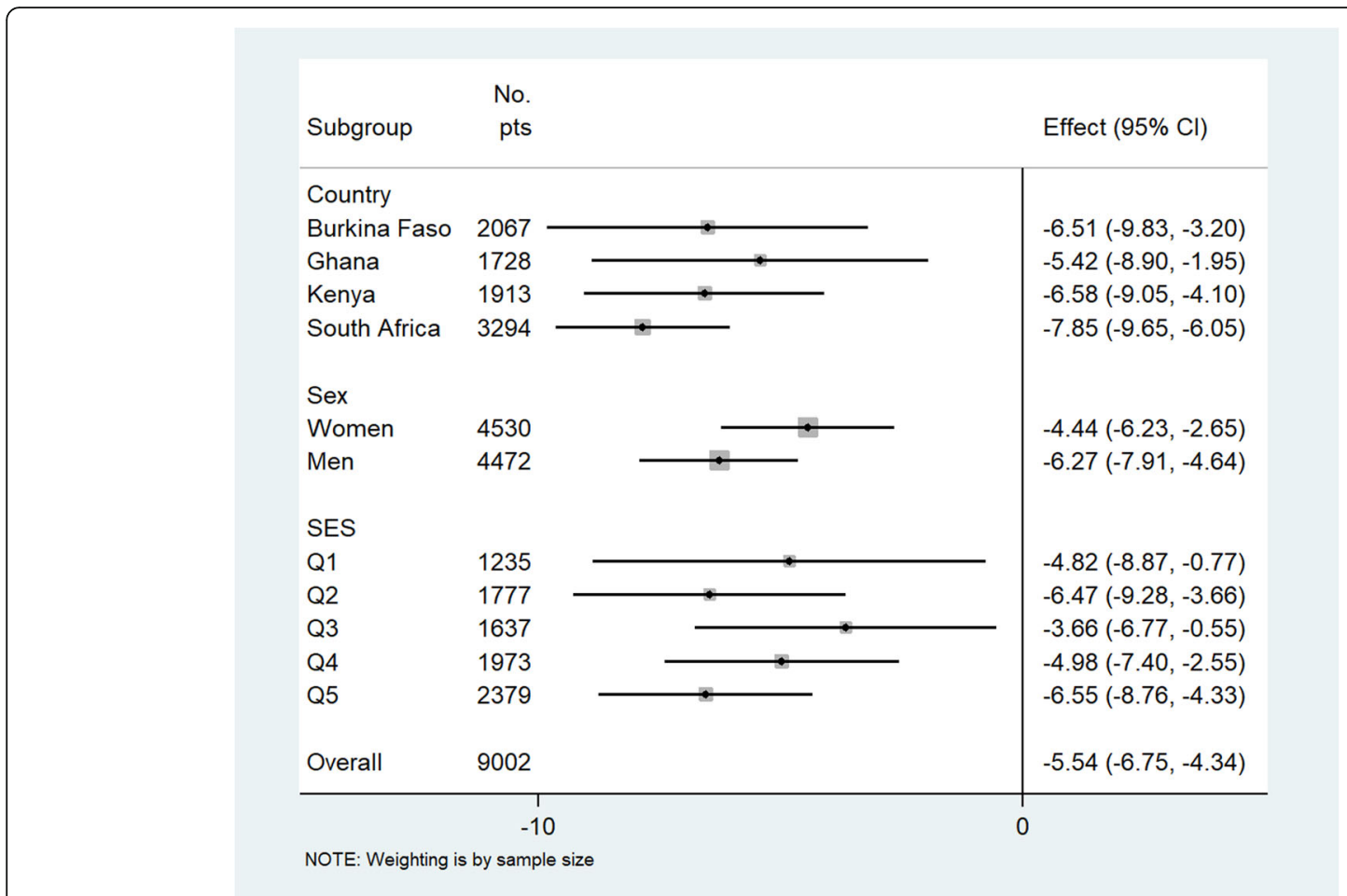

Fig. 2 Adjusted association of cardiovascular health index (CVHI) score with common carotid intima-media thickness (CIMT in $\mu \mathrm{m})$ in the combined AWI-Gen population stratified by country, sex, and socio-economic status (SES) subgroups. Effect is represented by $\beta$-coefficients and is a change in mean CIMT (in micrometers) with each unit increase in the CVHI score (0-14). Wealth quintile defined as Q1 = poorest, Q2 = poorer, Q3 = poor, Q4 less poor, and Q5 = least poor. Adjusted for age, educational status, household SES, HIV + ART, self-reported history of stroke, heart attack, congestive heart failure, and chronic kidney disease

Gen participants into the ideal diet category. This notwithstanding, the broad range of $\mathrm{iCVH}$ levels observed in the various settings may be a reflection of the level of ongoing epidemiological transition from communicable to non-communicable diseases. Thus, the $\mathrm{iCVH}$ levels were much higher in rural communities in Ghana and Burkina Faso and lower in the more urban settings of Kenya and South Africa and lowest in Europe and the USA.

We observed an inverse association of the $\mathrm{iCVH}$ with common CIMT levels independent of age, educational status, socio-economic status, HIV infection, and ART use. Similar to our findings, several other studies conducted in Caucasian, African-American, and Asian populations have observed increasing CVHI score is associated with lower risk of subclinical atherosclerosis $[15,17,31-35]$. It has been reported that a proportion of residual phenotypic variance is due to the additive effects of genes [36]. Although we did not adjust for the effect of genes in this paper, the Emory Twin Study did report that shared genetic and other familial factors do not confound the association of CVHI with CIMT [15]. Our results did not show any significant disparities in the association between CVHI and CIMT across different levels of socio-economic wealth quintiles as has been previously reported in a multi-ethnic population, including
African Americans, in the USA [25]. This may be due to the fact that participants in our population did not show significant difference in wealth distribution.

The rising burden of CVD morbidity and mortality in SSA has been reported to occur in younger age groups $[1,37]$. This, coupled with high levels of unawareness of risk factors and challenges with secondary prevention, calls for the evaluation of simple tools that can be used in routine screening for subjects with poor $\mathrm{CVH}$ in primary prevention efforts. The significant independent association of CIMT with the CVHI demonstrated in this study makes a strong case for the utility of the CVHI metric in the routine screening of CVD risk in the broader SSA population irrespective of SES status.

We observed in the combined sample that BMI, blood pressure, and hyperglycemia were the CVHI metrics most strongly associated with common CIMT. The former 2 components were also associated with common CIMT in both women and men while physical activity and smoking related with common CIMT in women but not men. This could be due to the fact that women were less likely to smoke compared to men and equally had healthier physical activity levels than men. These findings have implications for prioritizing particular CVHI components for the primary prevention of atherosclerotic cardiovascular diseases 


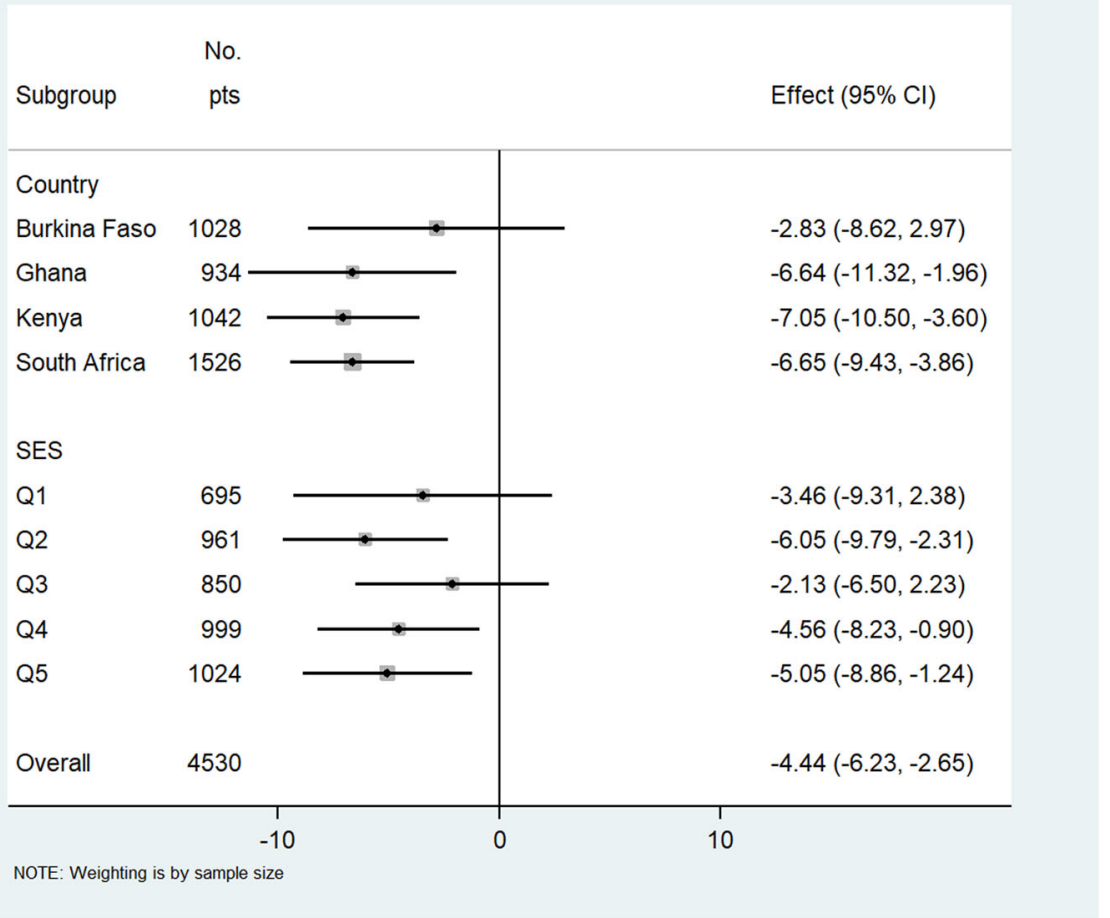

Fig. 3 Adjusted association of cardiovascular health index (CVHI) score with common carotid intima-media thickness (CIMT in $\mu \mathrm{m})$ in women stratified by country and socio-economic status (SES). Effect is represented by $\beta$-coefficients and is a change in mean CIMT (in micrometers) with each unit increase in the CVHI score (0-14). Wealth quintile defined as Q1 = poorest, Q2 = poorer, Q3 = poor, Q4 less poor, and Q5 = least poor. Adjusted for age, educational status, household SES, HIV + ART, self-reported history of stroke, heart attack, congestive heart failure, and chronic kidney disease

in SSA. Although total cholesterol did not correlate with CVHI, cholesterol levels in Africans remain lower than in the Western World. However, with urbanization, cholesterol levels are increasing and thus may well contribute in the near future, to atherosclerotic cardiovascular disease in SSA [38].

Our study does have some limitations. Reliance on self-reported smoking, physical activity, HIV infection status, and ART use may result in recall and social desirability biases. Diet was ascertained as self-reported servings of fruits and vegetables per day instead of derived from five components (fruits and vegetables per day; portions of fish a week; servings of fiber-rich whole grains per day; sodium per day and sugar-sweetened beverages per week) as recommended by AHA, which could have resulted in misclassification of certain participants in terms of their dietary intake. In addition, the cut points used for classifying each of the components of the CVHI metric were largely based on those used in populations of European ancestry. This brings into question whether these cut points are appropriate for use in African populations. However, the cut points used in the CVHI for defining cholesterol, blood pressure, glucose, and BMI levels are used globally for the categorization of these disease markers and are recognized cut points in the clinical guidelines of many African nations; their use within these countries has not been widely questioned. Other cut points have been challenged for their use within African populations and include those for HDL and triglycerides [39], HbA1c [40], and waist circumference [41], but none of these factors are used within the CVHI metric.

Another possible shortcoming of the use of the CVHI in African populations is the high prevalence of underweight which is classified in the ideal category for BMI but is associated with increased cardiovascular risk. However, a large meta-analysis has shown that low BMI is more strongly associated with respiratory disease than CVD mortality, but it must be noted that this review included few studies from Africa [42]. Future studies confirming the applicability of the CVHI criteria to African populations, especially those for BMI, would be important. In the current study, it was essential to use the existing CVHI criteria so that we were able to compare our data to those from studies conducted in both African and non-African populations.

Despite these limitations, the greatest strength of this work is that it used a large cohort of African individuals 


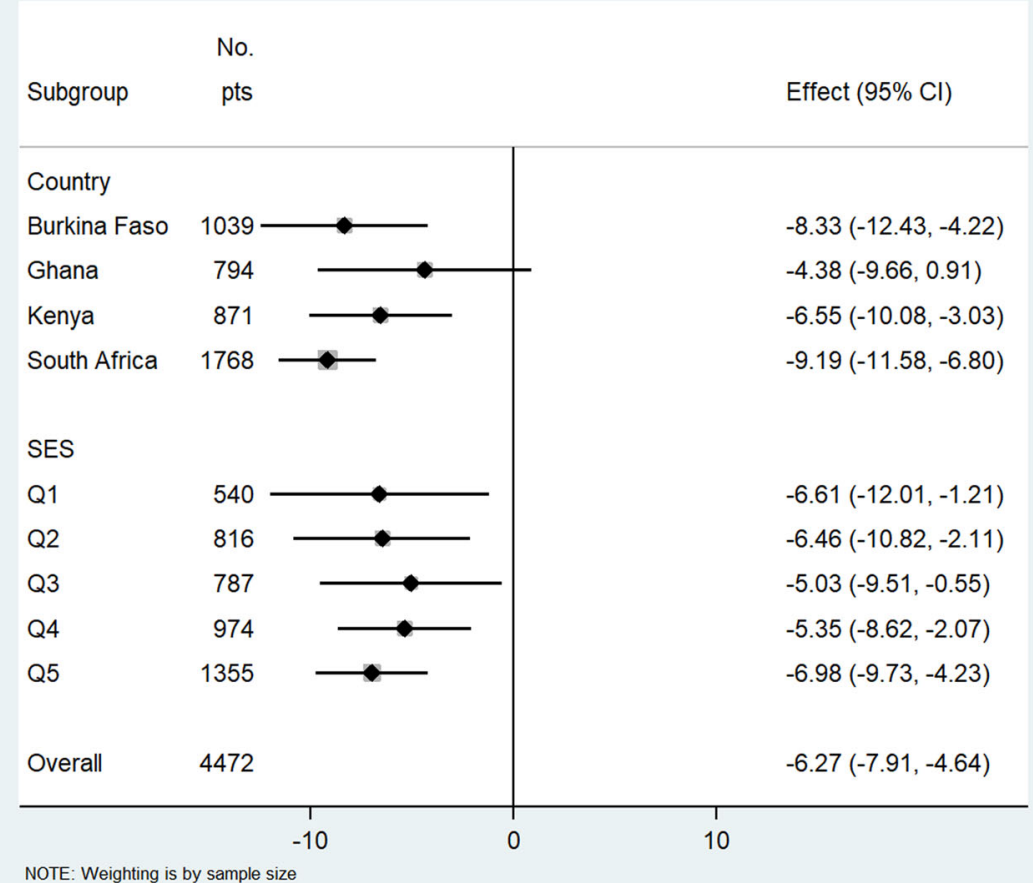

Fig. 4 Adjusted association of cardiovascular health index (CVHI) score with common carotid intima-media thickness (CIMT in micrometers) in men stratified by country and socio-economic status (SES). Effect is represented by $\beta$-coefficients and is a change in mean CIMT (in $\mu \mathrm{m}$ ) with each unit increase in the CVHI score (0-14). Wealth quintile defined as Q1 = poorest, Q2 = poorer, Q3 = poor, Q4 less poor, and Q5 = least poor. Adjusted for age, educational status, household SES, HIV + ART, self-reported history of stroke, heart attack, congestive heart failure, and chronic kidney disease

Table 3 Association of CVHI score with common CIMT ( $\mu \mathrm{m})$ in women and men in the combined AWI-Gen sample and by countries

\begin{tabular}{|c|c|c|c|c|c|}
\hline & $\begin{array}{l}\text { All countries } \\
\beta[95 \% \mathrm{Cl}]\end{array}$ & $\begin{array}{l}\text { Burkina Faso } \\
\beta[95 \% \mathrm{Cl}]\end{array}$ & $\begin{array}{l}\text { Ghana } \\
\beta[95 \% \mathrm{Cl}]\end{array}$ & $\begin{array}{l}\text { Kenya } \\
\beta[95 \% \mathrm{Cl}]\end{array}$ & $\begin{array}{l}\text { South Africa } \\
\beta[95 \% \mathrm{Cl}]\end{array}$ \\
\hline \multicolumn{6}{|c|}{ Combined sample } \\
\hline Model 1 & $-7.39[-8.49,-6.27]$ & $-6.48[-9.80,-3.16]$ & $-5.42[-8.89,-1.94]$ & $-6.79[-9.24,-4.35]$ & $-8.31[-10.1,-6.52]$ \\
\hline Model 2 & $-7.15[-7.89,-6.41]$ & $-6.43[-9.76,-3.09]$ & $-5.46[-8.94,-1.97]$ & $-6.66[-9.12,-4.19]$ & $-7.66[-9.47,-5.85]$ \\
\hline Model 3 & $-7.22[-8.04,-6.39]$ & $-6.51[-9.83,-3.19]$ & $-5.42[-8.91,-1.94]$ & $-6.57[-9.05,-4.10]$ & $-7.85[-9.65,-6.05]$ \\
\hline Model 4 & $-7.11[-7.74,-6.48]$ & $-6.42[-9.75,-3.09]$ & $-5.51[-8.99,-2.02]$ & $-6.63[-9.10,-4.16]$ & $-7.49[-9.31,-5.68]$ \\
\hline \multicolumn{6}{|l|}{ Women } \\
\hline Model 1 & $-5.98[-7.44,-4.52]$ & $-2.05[-7.73,3.62]$ & $-6.04[-10.60,-1.46]$ & $-6.81[-10.2,-3.43]$ & $-6.89[-9.69,-4.10]$ \\
\hline Model 2 & $-5.85[-7.00,-4.71]$ & $-2.18[-7.98,3.62]$ & $-6.59[-11.20,-1.96]$ & $-7.05[-10.50,-3.64]$ & $-6.62[-9.39,-3.86]$ \\
\hline Model 3 & $-4.87[-5.69,-4.05]$ & $-2.83[-8.63,2.97]$ & $-6.64[-11.30,-1.96]$ & $-7.05[-10.50,-3.59]$ & $-6.65[-9.44,-3.86]$ \\
\hline Model 4 & $-5.93[-7.02,-4.85]$ & $-2.29[-8.10,3.53]$ & $-6.79[-11.40,-2.15]$ & $-7.16[-10.60,-3.71]$ & $-6.52[-9.34,-3.69]$ \\
\hline \multicolumn{6}{|l|}{ Men } \\
\hline Model 1 & $-7.83[-9.79,-5.86]$ & $-8.62[-12.7,-4.52]$ & $-4.69[-10.1,0.66]$ & $-6.66[-10.20,-3.15]$ & $-9.36[-11.70,-7.01]$ \\
\hline Model 2 & $-7.42[-9.21,-5.63]$ & $-8.25[-12.4,-4.14]$ & $-4.56[-9.91,0.79]$ & $-6.51[-10.00,-2.99]$ & $-8.84[-11.20,-6.44]$ \\
\hline Model 3 & $-5.68[-6.57,-4.80]$ & $-8.33[-12.4,-4.22]$ & $-4.38[-9.67,0.92]$ & $-6.55[-10.10,-3.02]$ & $-9.19[-11.60,-6.80]$ \\
\hline Model 4 & $-7.38[-9.11,-5.66]$ & $-8.18[-12.3,-4.07]$ & $-4.47[-9.81,0.87]$ & $-6.42[-9.93,-2.91]$ & $-8.71[-11.10,-6.32]$ \\
\hline
\end{tabular}

Effect measured as $\beta$-coefficient (change in mean CIMT in $\mu \mathrm{m}$ per unit change in CVHI score); model 1 adjusted for age and sex; model 2 adjusted for age, sex, education status, household SES, and site; model 3 adjusted for self-reported history of stroke, heart attack, congestive heart failure, and chronic kidney disease in addition to model 2; model 4 was adjusted for model 2 plus chronic kidney disease and HIV plus antiretroviral therapy (ART) use; model 1 for women and men were adjusted for only age. CVHI cardiovascular health index, CIMT carotid intima-media thickness 
Table 4 Adjusted association of components of the CVHI metric with common CIMT in the AWI-Gen population stratified by sex

\begin{tabular}{|c|c|c|c|c|c|c|c|}
\hline \multicolumn{2}{|l|}{ CVHI metrics } & \multicolumn{2}{|l|}{ All countries } & \multicolumn{2}{|l|}{ Women } & \multicolumn{2}{|l|}{ Men } \\
\hline & Levels & $\beta[95 \% \mathrm{Cl}]$ & $p$ value & $\beta[95 \% \mathrm{Cl}]$ & $p$ value & $\beta[95 \% \mathrm{Cl}]$ & $p$ value \\
\hline \multirow[t]{3}{*}{ Smoking } & Poor & Ref & Ref & Ref & Ref & Ref & Ref \\
\hline & Intermediate & $-1.01[-13.7,11.7]$ & 0.083 & $-11.4[-23.9,1.15]$ & $<0.001$ & $1.41[-12.9,15.7]$ & 0.658 \\
\hline & Ideal & $3.28[-0.09,7.35]$ & & $-7.04[-10.1,-3.98]$ & & $3.83[-16.7,9.04]$ & \\
\hline \multirow[t]{3}{*}{ Physical activity } & Poor & Ref & Ref & Ref & Ref & Ref & Ref \\
\hline & Intermediate & $-17.5[-34.9,-0.08]$ & 0.078 & $-19.8[-34.0,-5.59]$ & $<0.001$ & $-10.8[-34.2,12.6]$ & 0.365 \\
\hline & Ideal & $-12.8[-24.8,-0,78]$ & & $-9.28[-14.1,-4.51]$ & & $-12.63[-30.4,5.14]$ & \\
\hline \multirow[t]{3}{*}{ Diet } & Poor & Ref & Ref & Ref & Ref & Ref & Ref \\
\hline & Intermediate & $1.92[-4.24,8.09]$ & 0.169 & $5.68[-1.91,13.3]$ & 0.059 & $-2.47[-13.3,8.33]$ & 0.654 \\
\hline & Ideal & $6.75[-0.24,13.7]$ & & $7.55[-1.08,16.2]$ & & $6.11[0.32,11.9]$ & \\
\hline \multirow[t]{3}{*}{ Body mass index } & Poor & Ref & Ref & Ref & Ref & Ref & Ref \\
\hline & Intermediate & $-4.06[-17.4,-2.96]$ & $<0.001$ & $-13.2[-25.5,-0.94]$ & $<0.001$ & $2.57[-20.4,25.6]$ & $<0.001$ \\
\hline & Ideal & $-28.2[-44.2,-12.2]$ & & $-33.6[-50.6,-16.6]$ & & $-32.7[-55.1,-10.4]$ & \\
\hline \multirow[t]{3}{*}{ Hypertension } & Poor & Ref & Ref & Ref & Ref & Ref & Ref \\
\hline & Intermediate & $-17.7[-26.0,-9.28]$ & $<0.001$ & $-7.30[-20.4,-5.75]$ & $<0.001$ & $-23.8[-27.0,-20.6]$ & $<0.001$ \\
\hline & Ideal & $-31.4[-42.4,-20.3]$ & & $-20.2[-39.1,-1.28]$ & & $-28.9[-38.1,-19.7]$ & \\
\hline \multirow[t]{3}{*}{ Fasting glucose } & Poor & Ref & Ref & Ref & Ref & Ref & Ref \\
\hline & Intermediate & $-18.2[-35.8,-0.68]$ & $<0.001$ & $-23.3[-36.8,-9.86]$ & $<0.001$ & $-8.79[-38.7,21.1]$ & 0.377 \\
\hline & Ideal & $-14.2[-32.8,-4.42]$ & & $-21.8[-35.2,-8.27]$ & & $-0.57[-29.1,27.9]$ & \\
\hline \multirow[t]{3}{*}{ Total cholesterol } & Poor & Ref & Ref & Ref & Ref & Ref & Ref \\
\hline & Intermediate & $-1.89[-28.3,24.5]$ & 0.958 & $5.45[-15.1,25.9]$ & 0.542 & $-15.8[-43.3,11.6]$ & 0.265 \\
\hline & Ideal & $-0.58[-28.6,27.5]$ & & $10.53[-11.2,32.2]$ & & $-17.8[-41.9,6.21]$ & \\
\hline
\end{tabular}

Adjusted for age, sex, educational status, and socio-economic status adjusted for in the combined population while age, educational status, and socio-economic status adjusted for in the sex stratified analyses. Effect is represented by $\beta$-coefficients and is a change in mean CIMT (in $\mu \mathrm{m}$ ) with each unit increase in the CVHI components. $\mathrm{CVHI}$ cardiovascular heath index

from 4 different SSA countries and a standardized data collection protocol, with laboratory assays performed at a single laboratory. In addition, a large number of possible confounding or modulatory variables were measured for use in the multiple regression models. Furthermore, this is the largest African study to use the CVHI to assess cardiovascular health and its association with common CIMT and provides important information on the utility of this metric for screening for individuals at high risk for CVD in resource-poor environments.

\section{Conclusion}

The CVHI score has a significant inverse, independent association with common CIMT in older adults across four sub-Saharan African countries. This makes a case for its use as a simple tool to monitor cardiovascular health at a population and clinical level in SSA. The current study demonstrates that primary prevention of atherosclerotic CVDs in SSA should focus on reducing smoking and obesity, improved control of hypertension and hyperglycemia, and higher levels of physical activity.

\section{Supplementary Information}

The online version contains supplementary material available at https://doi. org/10.1186/s12916-021-01909-6.

Additional file 1: Table S1. Scoring and definition of components of Cardiovascular Health Index.

Additional file 2: Figure S1. Kernel density of mean distribution number of ideal cardiovascular health metrics (0-14) by AWI-Gen study countries. Figure S2. Kernel density of mean CIMT distribution in $\mu \mathrm{m}$ by AWI-Gen study countries. Figure S3. The linear association between $\mathrm{CVH}$ score and CIMT in the combined AWI-Gen population. Figure S4. The linear association between $\mathrm{CVH}$ score and CIMT in the combined four participating countries of the AWI-Gen study.

\section{Abbreviations}

AHA/ASA: American Heart Association and the American Stroke Association; AWI-Gen: African Wits-INDEPTH Genomic studies; BMI: Body mass index; CVD: Cardiovascular disease; CIMT: Carotid-Intima media thickness;

CVHI: Cardiovascular Health Index; HDSS: Health and Socio-demographic Surveillance System; SSA: Sub-Saharan Africa

\section{Acknowledgements}

We are grateful to the participants for their generosity and sacrifice for spending many hours responding to questionnaires, being measured, and having samples taken. We wish to acknowledge the sterling contributions of our field workers, phlebotomists, laboratory scientists, administrators, data personnel, and other staff 
who contributed to the data and sample collections, processing, storage, and shipping.

\section{Authors' contributions}

EAN, NJC, MLB, and KKG conceived the study and designed the analysis plan. EAN conducted the literature search, analyzed the data, and wrote the first draft. MLB, NJC, and KKG contributed to revising the text and writing the paper. EAN, NJC, ARO, GA, LKM, SFM, SSRC, PRB, HS, SMT, SAN, DEG, FJR, $M L B, M R$, and $K K G$ critically reviewed the paper. $M R$ is overall principal investigator; $A R O, S M T$, and SAN are site principal investigators of the AWIGen study and together with sites investigators EAN, NJC, ARO, GA, LKM, SFM, PRB, HS, SMT, and SAN acquired data and had data oversight. All authors reviewed and approved the manuscript before submitting for publication.

\section{Funding}

The AWI-Gen Collaborative Centre is funded by the National Human Genome Research Institute (NHGRI), Office of the Director (OD), Eunice Kennedy Shriver National Institute Of Child Health \& Human Development (NICHD), the National Institute of Environmental Health Sciences (NIEHS), the Office of AIDS Research (OAR), and the National Institute of Diabetes and Digestive and Kidney Diseases (NIDDK), of the National Institutes of Health under award number U54HG006938 and its supplements, as part of the H3Africa Consortium. Additional funding was leveraged from the Department of Science and Technology, South Africa, award number DST/CON 0056/2014. EAN receives support from the Global Health Support Program of the University Medical Center Utrecht (UMCU), Utrecht University, the Netherlands, and the Navrongo Health Research Centre (NHRC), Ghana.

\section{Availability of data and materials}

The datasets generated and/or analyzed during the current study will be made publicly available in the European Genome-phenome Archive under the set of projects related to the Human Heredity and Health in Africa (H3Africa) Consortium. Details concerning access to data and DNA can be found in the document titled H3Africa Data and Biospecimen Access Committee Guidelines, available in the consortium documents section of the H3Africa website (www.h3africa.com).

\section{Ethics approval and consent to participate}

This study received ethical approval from the Human Research Ethics Committee (Medical) of the University of the Witwatersrand (Wits) (protocol number M121029) and was renewed in 2017 (protocol number M170880). Additional approvals were obtained from the local ethics review boards in research site prior to commencing any participant-related activities.

\section{Consent for publication}

Not applicable

\section{Competing interests}

The authors declare that they have no competing interests.

\section{Author details}

${ }^{1}$ Clinical Sciences Department, Navrongo Health Research Centre, Ghana Health Service, Navrongo, Ghana. ${ }^{2} J u l i u s$ Global Health, Julius Center for Health Sciences and Primary Care, University Medical Center Utrecht, Utrecht University, Utrecht, the Netherlands. ${ }^{3}$ Department of Chemical Pathology, National Health Laboratory Service, Faculty of Health Services, University of the Witwatersrand, Johannesburg, South Africa. ${ }^{4} \mathrm{MRC}$ Wits Developmental Pathways for Health Research Unit, Faculty of Health Sciences, University of the Witwatersrand, Johannesburg, South Africa. ${ }^{5}$ Institut de Recherché en Sciences de la Santé, Clinical Research Unit of Nanoro, Nanoro, Burkina Faso. ${ }^{6}$ DIMAMO Health Demographic Surveillance Site, Department of Pathology and Medical Sciences, School of Health Care Sciences, Faculty of Health Sciences, University of Limpopo, Polokwane, South Africa. ${ }^{7}$ African Population Health Research Centre, Nairobi, Kenya. ${ }^{8}$ MRC/Wits Rural Public Health and Health Transitions Research Unit, School of Public Health, Faculty of Health Sciences, University of the Witwatersrand, Johannesburg, South Africa. ${ }^{9}$ Carbohydrate and Lipid Metabolism Research Unit, Faculty of Health Sciences, University of the Witwatersrand, Johannesburg, South Africa. ${ }^{10}$ Sydney Brenner Institute of Molecular Bioscience, Faculty of Health Sciences, University of the Witwatersrand, Johannesburg, South Africa. ${ }^{11}$ Division of Epidemiology and Biostatistics, School of Public Health, Faculty of Health Sciences, University of the Witwatersrand, Johannesburg, South Africa.
Received: 16 October 2020 Accepted: 13 January 2021 Published online: 10 February 2021

\section{References}

1. GBD 2015 Risk Factors Collaborators. Global, regional, and national comparative risk assessment of 79 behavioural, environmental and occupational, and metabolic risks or clusters of risks, 1990-2015: a systematic analysis for the Global Burden of Disease Study 2015. Lancet. 2016;388(10053):1659-724.

2. Roth GA, Johnson C, Abajobir A, Abd-Allah F, Abera SF, Abyu G, et al. Global, regional, and national burden of cardiovascular diseases for 10 causes, 1990 to 2015. J Am Coll Cardiol. 2017;70(1):1-25.

3. Santosa A, Byass P. Diverse empirical evidence on epidemiological transition in low- and middle-income countries: population-based findings from INDE PTH network data. PLoS One. 2016;11(5):e0155753.

4. Bawah A, Houle B, Alam N, Razzaque A, Streatfield PK, Debpuur C, et al. The evolving demographic and health transition in four low- and middleincome countries: evidence from four sites in the INDEPTH network of longitudinal health and demographic surveillance systems. Plos One. 2016; 11(6):e0157281.

5. D'Agostino RB Sr, Vasan RS, Pencina MJ, Wolf PA, Cobain M, Massaro JM, et al. General cardiovascular risk profile for use in primary care: the Framingham Heart Study. Circulation. 2008;117(6):743-53.

6. Goff DC Jr, Lloyd-Jones DM, Bennett G, Coady S, D'Agostino RB Sr, Gibbons R, et al. 2013 ACC/AHA guideline on the assessment of cardiovascular risk: a report of the American College of Cardiology/ American Heart Association Task Force on Practice Guidelines. J Am Coll Cardiol. 2014;63(25 Pt B):2935-59.

7. Boateng D, Agyemang C, Beune E, Meeks K, Smeeth L, Schulze MB, et al. Cardiovascular disease risk prediction in sub-Saharan African populations comparative analysis of risk algorithms in the RODAM study. Int J Cardiol. 2018;254:310-5.

8. Lloyd-Jones DM, Hong Y, Labarthe D, Mozaffarian D, Appel LJ, Van Horn L, et al. Defining and setting national goals for cardiovascular health promotion and disease reduction: the American Heart Association's strategic Impact Goal through 2020 and beyond. Circulation. 2010;121(4): 586-613.

9. Shay CM, Gooding HS, Murillo R, Foraker R. Understanding and improving cardiovascular health: an update on the American Heart Association's concept of cardiovascular health. Prog Cardiovasc Dis. 2015;58(1):41-9.

10. Shay CM, Ning H, Daniels SR, Rooks CR, Gidding SS, Lloyd-Jones DM. Status of cardiovascular health in US adolescents: prevalence estimates from the National Health and Nutrition Examination Surveys (NHANES) 2005-2010. Circulation. 2013;127(13):1369-76.

11. Huffman MD, Capewell S, Ning H, Shay CM, Ford ES, Lloyd-Jones DM. Cardiovascular health behavior and health factor changes (1988-2008) and projections to 2020: results from the National Health and Nutrition Examination Surveys. Circulation. 2012;125(21):2595-602.

12. Touboul PJ, Grobbee DE, den Ruijter H. Assessment of subclinical atherosclerosis by carotid intima media thickness: technical issues. Eur J Prev Cardiol. 2012;19(2 Suppl):18-24.

13. Bots ML, Evans GW, Tegeler CH, Meijer R. Carotid intima-media thickness measurements: relations with atherosclerosis, risk of cardiovascular disease and application in randomized controlled trials. Chin Med J. 2016;129(2): 215-26.

14. Nonterah EA, Boua PR, Klipstein-Grobusch K, Asiki G, Micklesfield LK, Agongo G, et al. Classical cardiovascular risk factors and HIV are associated with carotid intima-media thickness in adults from Sub-Saharan Africa: Findings From H3Africa AWI-Gen Study. J Am Heart Assoc. 2019;8(14): e011506.

15. Kulshreshtha A, Goyal A, Veledar E, McClellan W, Judd S, Eufinger SC, et al. Association between ideal cardiovascular health and carotid intima-media thickness: a twin study. J Am Heart Assoc. 2014;3(1):e000282.

16. Saleem Y, DeFina LF, Radford NB, Willis BL, Barlow CE, Gibbons LW, et al. Association of a favorable cardiovascular health profile with the presence of coronary artery calcification. Circ Cardiovasc Imaging. 2015;8(1):e001851.

17. Younus A, Aneni EC, Spatz ES, Osondu CU, Roberson L, Ogunmoroti O, et al. A systematic review of the prevalence and outcomes of ideal cardiovascular health in US and non-US populations. Mayo Clin Proc. 2016;91(5):649-70.

18. Feinstein MJ, Kim JH, Bibangambah P, Sentongo R, Martin JN, Tsai AC, et al. Ideal cardiovascular health and carotid atherosclerosis in a mixed cohort of 
HIV-infected and uninfected Ugandans. AIDS Res Hum Retrovir. 2017;33(1): 49-56.

19. Ketelaar EJ, Vos AG, Godijk NG, Scheuermaier K, Devillé W, Tempelman H, et al. Ideal cardiovascular health index and its determinants in a rural South African population. Global Heart. 2020;15(1):76.

20. Ramsay M, Crowther NJ, Tambo E, Agongo G, Baloyi V, Dikotope S, et al. H3Africa AWI-Gen Collaborative Centre: a resource to study the interplay between genomic and environmental risk factors for cardiometabolic diseases in four sub-Saharan African countries. Glob Health Epidemiol Genomics. 2016;1(e20):1-13.

21. Ali SA, Soo C, Agongo G, Alberts M, Amenga-Etego L, Boua RP, et al. Genomic and environmental risk factors for cardiometabolic diseases in Africa: methods used for Phase 1 of the AWI-Gen population cross-sectional study. Glob Health Action. 2018;11(sup2):1507133.

22. WHO. Global recommendations on physical activity for health. Geneva: World Health Organisation; 2010.

23. Ferro-Luzzi A, Garza C, Haas J, Habicht JP, Himes J, Pradilla A, et al. Physical status: the use and interpretation of anthropometry. Geneva: World Health Organisation; 1995

24. Klug E, Raal FJ, Marais AD, Taskinen M-R, Dalby AJ, Schamroth C, et al. South African dyslipidaemia guideline consensus statement. S Afr Med J. 2012; 102(3 Pt 2):178-87.

25. Polonsky TS, Ning H, Daviglus ML, Liu K, Burke GL, Cushman M, et al. Association of cardiovascular health with subclinical disease and incident events: the multi-ethnic study of atherosclerosis. J Am Heart Assoc. 2017;6(3): e004894.

26. Shea S, Lima J, Diez-Roux A, Jorgensen NW, McClelland RL. Socioeconomic status and poor health outcome at 10 years of follow-up in the multi-ethnic study of atherosclerosis. PLoS One. 2016;11(11):e0165651.

27. Rundek T, Brown DL. Socioeconomic status and subclinical atherosclerosis: are we closing disparity gaps? Stroke. 2014;45(4):948-9.

28. Pilkerton CS, Singh SS, Bias TK, Frisbee SJ. Changes in cardiovascular health in the United States, 2003-2011. J Am Heart Assoc. 2015;4(9):e001650.

29. van Nieuwenhuizen B, Zafarmand MH, Beune E, Meeks K, Aikins AD, Addo J, et al. Ideal cardiovascular health among Ghanaian populations in three European countries and rural and urban Ghana: the RODAM study. Intern Emerg Med. 2018;13(6):845-56.

30. Peng Y, Cao S, Yao Z, Wang Z. Prevalence of the cardiovascular health status in adults: a systematic review and meta-analysis. Nutr Metab Cardiovasc Dis. 2018;28(12):1197-207.

31. Xanthakis V, Enserro DM, Murabito JM, Polak JF, Wollert KC, Januzzi JL, et al. Ideal cardiovascular health: associations with biomarkers and subclinical disease and impact on incidence of cardiovascular disease in the Framingham Offspring Study. Circulation. 2014;130(19):1676-83.

32. Shpilsky D, Bambs C, Kip K, Patel S, Aiyer A, Olafiranye O, et al. Association between ideal cardiovascular health and markers of subclinical cardiovascular disease. Clin Cardiol. 2018:41(12):1593-9.

33. Singh SS, Pilkerton CS, Shrader CD Jr, Frisbee SJ. Subclinical atherosclerosis, cardiovascular health, and disease risk: is there a case for the Cardiovascular Health Index in the primary prevention population? BMC Public Health. 2018;18(1):429

34. Santos IS, Goulart AC, Pereira AC, Lotufo PA, Bensenor IM. Association between cardiovascular health score and carotid intima-media thickness: cross-sectional analysis of the Brazilian Longitudinal Study of Adult Health (ELSA-Brasil) baseline assessment. J Am Soc Echocardiogr. 2016;29(12):1207-16 e4.

35. Gao J, Bao M, Liu Y, Shi J, Huang Z, Xing A, et al. Changes in cardiovascular health score and atherosclerosis progression in middle-aged and older persons in China: a cohort study. BMJ Open. 2015;5(8):e007547.

36. Kathiresan S, Srivastava D. Genetics of human cardiovascular disease. Cell. 2012;148(6):1242-57.

37. Yuyun MF, Sliwa K, Kengne AP, Mocumbi AO, Bukhman G. Cardiovascular diseases in Sub-Saharan Africa compared to high-income countries: an epidemiological perspective. Global Heart. 2020;15(1):15.

38. Noubiap JJ, Bigna JJ, Nansseu JR, Nyaga UF, Balti EV, Echouffo-Tcheugui JB, et al. Prevalence of dyslipidaemia among adults in Africa: a systematic review and meta-analysis. Lancet Glob Health. 2018;6(9):e998-e1007.

39. Bentley AR, Rotimi CN. Interethnic differences in serum lipids and implications for cardiometabolic disease risk in African ancestry populations. Glob Heart. 2017;12(2):141-50.
40. Zemlin AE, Matsha TE, Hassan MS, Erasmus RT. HbA1c of $6.5 \%$ to diagnose diabetes mellitus -- does it work for us? -- the Bellville South Africa study. Plos One. 2011;6(8):e22558.

41. Ekoru K, Murphy GAV, Young EH, Delisle H, Jerome CS, Assah F, et al. Deriving an optimal threshold of waist circumference for detecting cardiometabolic risk in sub-Saharan Africa. Int J Obes. 2017:42(3):487-94.

42. Di Angelantonio E, Bhupathiraju SN, Wormser D, Gao P, Kaptoge S, de Gonzalez $A B$, et al. Body-mass index and all-cause mortality: individualparticipant-data meta-analysis of 239 prospective studies in four continents. Lancet. 2016;388(10046):776-86.

\section{Publisher's Note}

Springer Nature remains neutral with regard to jurisdictional claims in published maps and institutional affiliations.

\section{Ready to submit your research? Choose BMC and benefit from:}

- fast, convenient online submission

- thorough peer review by experienced researchers in your field

- rapid publication on acceptance

- support for research data, including large and complex data types

- gold Open Access which fosters wider collaboration and increased citations

- maximum visibility for your research: over $100 \mathrm{M}$ website views per year

At BMC, research is always in progress.

Learn more biomedcentral.com/submissions 